\title{
PIELONEFRITIS ENFISEMATOSA: PRESENTACIÓN DE UN CASO Y REVISIÓN DE LA LITERATURA
}

\author{
A. BLANCO DÍEZ, A. BARBAGELATA LÓPEZ, E. FERNÁNDEZ ROSADO, R. CASAS \\ MUÍÑO, V. CHANTADA ABAL, M. GONZÁLEZ MARTÍN.
}

Servicio de Urología. Hospital Universitario Juan Canalejo. La Coruña.

Actas Urol Esp. 27 (9): 721-725, 2003

\section{RESUMEN}

PIELONEFRITIS ENFISEMATOSA: PRESENTACIÓN DE UN CASO Y REVISIÓN DE LA LITERATURA

La pielonefritis enfisematosa es una infección necrotizante del parénquima renal que afecta especialmente a pacientes diabéticos con mal control de su enfermedad. Esta grave afección conlleva altas tasas de mortalidad aún a pesar del tratamiento agresivo. Lo más importante en el manejo de la misma es un precoz diagnóstico y agresivo tratamiento, casi siempre con nefrectomía.

Presentamos un caso clínico típico, haciendo especial hincapié en el diagnóstico clínico y por imagen, así como en el tratamiento, y en su evolución. Asimismo realizamos una revisión de la literatura intentando comprender mejor este proceso, y encontrar bases definidas para su abordaje terapéutico.

PALABRAS CLAVE: Pielonefritis enfisematosa. Diagnóstico. Tratamiento.

\section{ABSTRACT}

EMPHYSEMATOUS PYELONEPHRITIS: CASE REPORT AND LITERATURE REVIEW.

Emphysematous pyelonephritis is a necrotizing renal parenquima infection, wich is diabetes mellitus related in almost all cases. This is life threatening and has a high mortality rates despite agressive management. The most important thing in management is a high diagnostic suspicious and also a rapid treatment, wich means nephrectomy.

We expose a case giving especial importance to clinical and image diagnose as treatment and evolution. We also make a literature review trying to understand this entity and find pathways for its therapeutic management.

KEY WORDS: Emphysematous pyelonephritis. Diagnose. Management.

$\mathrm{L}^{\mathrm{a}}$ a pielonefritis enfisematosa es una grave afectación aguda y necrotizante del parénquima renal y tejidos perirrenales, causada por uropatógenos gram-negativos que se comportan como anaerobios facultativos en determinadas situaciones de baja concentración de oxígeno, creando una infección y produciendo gas por mecanismos aún no del todo esclarecidos. Es más frecuente en mujeres y diabéticos, asociado o no a obstrucción o factor estásico ureteral. La evolución sin tratamiento, o con tratamiento médico únicamente alcanza tasas de mortalidad del $80 \%$. Esto último hace que el diagnóstico rápido, sospechándolo en un diabético con foco séptico urinario, o no conocido, con empeoramiento de su estado general y escasa respuesta a antibióticos convencionales, sea esencial en aras de un manejo agresivo y precoz que garantice mayores posibilidades de éxito. Los datos clínicos y de laboratorio raramente conducen al diagnóstico, siendo imprescindibles estudios radiológicos. 
En este artículo se aporta un caso más a la escasa casuística existente, que es típico en su desarrollo, evolución y manejo, y que pone de manifiesto la necesidad de la sospecha precoz, los datos radiológicos, y el tratamiento agresivo de entrada.

\section{CASO CLÍNICO}

Paciente varón de 31 años con antecedentes personales de: fumador de un paquete/día, tuberculosis pulmonar en la infancia, DMID (a tratamiento con insulina NPH 36-0-26), accidente de tráfico en el 95 con necesidad de intervención quirúrgica por rotura hepática.

Acude al servicio de urgencias relatando que 15 días antes había iniciado un cuadro de polaquiuria y disuria que lo llevó a su médico de cabecera, le diagnostica infección urinaria y le pauta ciclo corto de antibiótico. 7 días después y una vez finalizado el tratamiento vuelve a acudir a su médico por iniciar un dolor en región lumbar izquierda de naturaleza constante y exacerbación con los movimientos, etiquetándose de proceso mecánico y tratado con miorrelajantes.

A los cuatro días de haber iniciado el nuevo tratamiento sufre un empeoramiento progresivo en su estado general acompañado de náuseas y vómitos de contenido alimenticio, con escalofríos, tiritona y disuria. Ante lo cual el paciente decide abandonar el tratamiento con insulina.

Finalmente ante la mala evolución (aumento de dolor, somnolencia...) el paciente acude a urgencias. A su llegada se detecta un importante descontrol glucémico (Bm test: > 500).

La exploración física revela: $\mathrm{T}^{\mathrm{a}}$ : $37^{\circ} \mathrm{C}$. Fc: 100 1.p.m. T.A.:118/80. Se encuentra consciente, somnoliento, aceptablemente colaborador y orientado en los tres ejes. Desde el punto de vista neurológico no se aprecia focalidad, ni signos meníngeos y las pupilas son isocóricas y normorreactivas. La auscultación cardiopulmonar se caracteriza por la presencia de una taquipnea (35 resp/minuto) y roncus aislados en ambos campos. A nivel de cabeza y cuello se aprecia facies sudorosa, lengua seca, signo del pliegue positivo. En la región abdominal presenta una cicatriz de laparotomía combinada (media y transversal), un abdomen blando y depresible, no doloroso, no defensa, no datos de peritonismo, RHA +, la puño-percusión es positiva en fosa lumbar izquierda.

Los datos analíticos muestran los siguientes resultados: Hemograma: Hto 47\%, leucocitos $22000 / \mathrm{mm} 3$, plaquetas 183.000. Coagulación:TP 17,10 seg. Bioquímica: urea $125 \mathrm{mgr} / \mathrm{dl}$, creatinina 3,5 mgr/dl, k+7,6 mEq/1, Na2+ $108 \mathrm{MEq} / 1$, glucosa $1430 \mathrm{mgr} / \mathrm{dl}$. Gasometría venosa: $\mathrm{Ph}$ 6,95, Hco3-4,3.

En la orina se aprecia: sedimento: 6-8 hematíes/campo, 20-25 leucos/campo, algunos gérmenes. Na2+ en orina $108 \mathrm{Meq} / 1 \mathrm{y} \mathrm{K}+$ de 14 MEq/1. Glucosuria y cetonuria:+.

Se enfoca de inicio como un cuadro de cetoacidosis diabética con infección urinaria acompañante con inestabilidad, por lo que se decide paso a UCI antes de completar estudios radiológicos.

En la UCI se inicia perfusión endovenosa continua con insulina, reposición hídrica, corrección de la acidosis y de los trastornos electrolíticos, comenzando con tratamiento antibiótico empírico de piperacilina-tazobactam + tobramicina. En la Rx simple de abdomen se aprecia un contorno aéreo delimitando la silueta renal izquierda (Fig. 1).

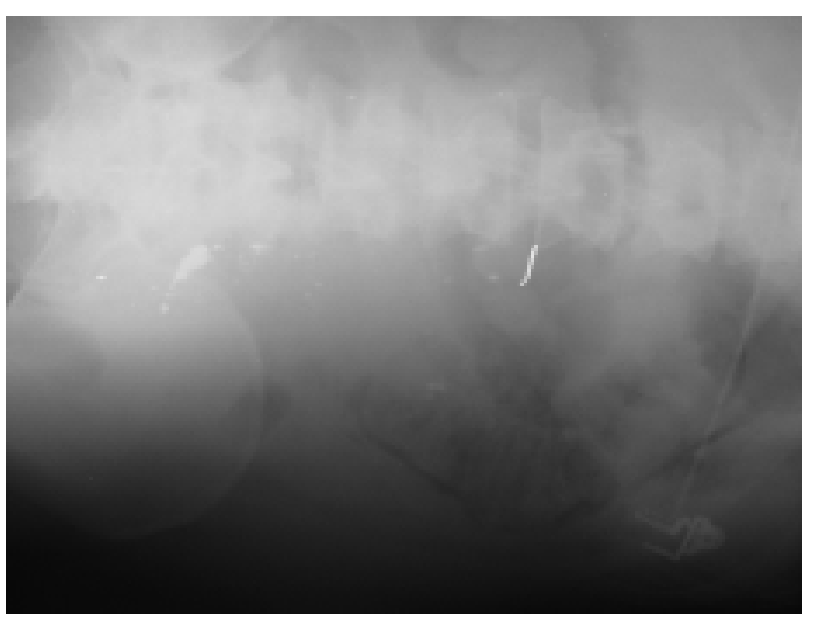

FIGURA 1. Radiografia simple de abdomen en la que podemos observar halo fino y oscuro que rodea la silueta renal izquierda, y que es compatible con la existencia de gas perirrenal.

Simultáneamente se practica ecografía abdominal que muestra importantes alteraciones del riñón izquierdo por lo que se decide practicar T.A.C. con resultado de imágenes compatibles con pielonefritis enfisematosa, que destruye de manera importante el parénquima del riñón izquierdo (Figs. 2 y 3). 


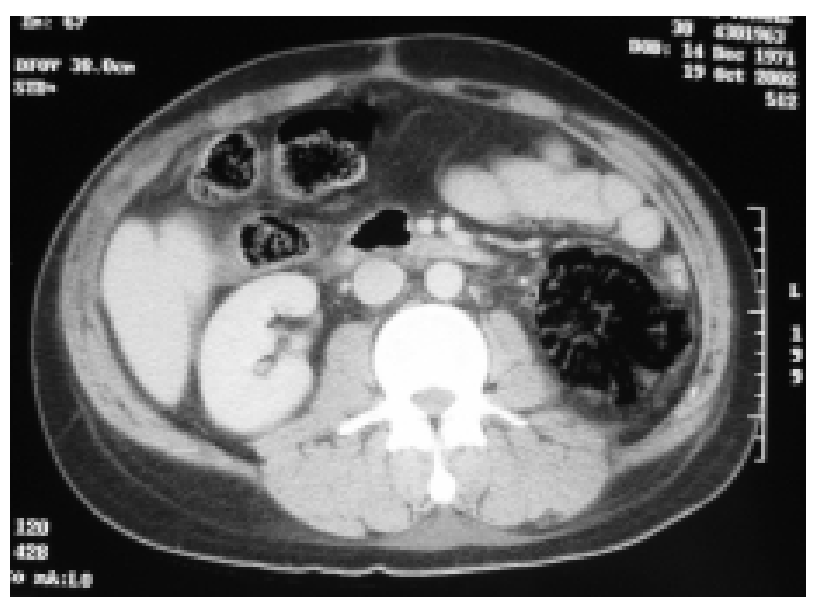

FIGURA 2. T.A.C. abdominal con contraste (corte a nivel de polo superior-medio del riñón izquierdo) en la que podemos observar destrucción parcial de parénquima, que es sustituido por imagen de densidad aire.



FIGURA 3. T.A.C. abdominal con contraste (corte a nivel de zona media del riñón) en la que podemos ver destrucción total del parénquima con ausencia del mismo y sustitución por material gaseoso.

Se diagnostica el cuadro como pielonefritis enfisematosa en paciente diabético descompensado y se decide intervención quirúrgica urgente.

Se realiza la incisión de lumbotomía, para evitar contaminación de cavidad peritoneal. Se observa grasa perirrenal edematosa, riñón con parcheado necrótico cortical irregular y cápsula conservada. Se liga pedículo en bloque extrayéndose la pieza.

Tras la cirugía el paciente retorna a la unidad de Cuidados Intensivos precisando de inicio ventilación mecánica no invasiva, en relación con una hipoxemia secundaria a aparición radiológica de infiltrado intersticial, que se interpreta como derivado de su situación de sepsis. Posteriormente mantiene una buena evolución con el devenir de las horas, recuperando su estabilidad hemodinámica y ventilatoria. La producción por drenajes es mínima y serosanguinolenta.

Se recibe el resultado de los hemocultivos y del cultivo de muestra tomada durante la intervención, coincidiendo ambos en el crecimiento de un E. Colli sensible a cefalosporinas de $3^{\mathrm{a}}$ generación, beta-lactámicos + inhibidores de las betalactamasas, aminoglucósidos, quinolonas y carbapenémicos. Dados los resultados y las concentraciones mínimas inhibitorias, se decide cesar la antibioterapia empírica cambiando a quinolonas (ciprofloxacino). Progresivamente se van recuperando parámetros analíticos de función renal y los trastornos hidroelectrolíticos. Es alta hospitalaria 11 días después de su ingreso tras ajuste de su insulinoterapia.

El análisis histológico de la pieza: extensas áreas de infarto hemorrágico; intensa pielonefritis aguda con formación de abscesos que se extienden a grasa perirrenal; trombos de fibrina en vasos.

\section{DISCUSIÓN}

Desde la primera aportación a la literatura de un caso de pielonefritis enfisematosa en 1898 por Kelly y Mac Callum ${ }^{1}$, existen alrededor de 100 publicados en el mundo, lo cual nos da indicios de la escasa incidencia de este cuadro. Sin embargo teniendo en cuenta la gravedad del mismo, la eventual dificultad diagnóstica si no se sospecha, y la rápida y mortal evolución, nos parece importante recordar ciertos aspectos. Hay que tener en cuenta que en lo relativo a la epidemiología se trata de un proceso, según se desprende de los datos aportados por las dos series más extensas de la literatura (Shekeir, 1997; Ahlering, 1985) $)^{2,3}$, cuya media de edad se sitúa en torno a los 55 años. Es más frecuente en mujeres (aprox. 75\%), y aunque puede ocurrir en pacientes no diabéticos, sobre todo asociado a obstrucción ureteral, el $80 \%$ los son, siendo aún más frecuente en diabetes mal controladas, con cierto grado de disfunción renal y/o necrosis papilar $^{2-6}$.

La patogenia está poco definida. Se piensa que a las áreas de microinfarto secundarias a microangiopatía diabética, con bajas tensiones de oxí- 
geno tisular, asociadas a altas concentraciones de glucosa en sangre por descompensaciones diabéticas se les suma la infección activa por uropatógenos habituales, los cuales en ausencia de oxígeno se convierten en anaerobios facultativos. Éstos utilizan la glucosa como sustrato alimentario, fermentándola y convirtiéndola en dióxido de carbono, produciendo gas ${ }^{4,7}$. En contra de lo que pudiera parecer la infección no es producida por anaerobios, sino por uropatógenos típicos, E. Coli (la gran mayoría), Klebsiella, Proteus, y menos frecuente por Pseudomonas ${ }^{2-7}$. En toda la literatura hay descrito un caso por Cándida ${ }^{8}$. Sólo existe un caso publicado de pielonefritis enfisematosa por anaerobios ${ }^{9}$. A pesar de su conocida propensión a formar gas el Clostridium no ha sido nunca aislado en este tipo de infecciones ${ }^{5}$.

La presentación clínica habitual, es la de una pielonefritis aguda severa que no responde a tratamiento. Casi todos los pacientes presentan la típica triada de fiebre, vómitos y dolor en flanco, asociados en ocasiones a masa en flanco. Este modo de presentación asociado a mal estado general, y descompensación diabética nos debe alertar siempre sobre la posibilidad de este cuadro. Los cultivos son positivos para el patógeno correspondiente.

El diagnóstico raramente se establece por la clínica y los datos de laboratorio (leucocitosis, a veces leucopenia, alargamiento de tiempos de coagulación, y demás signos de infección o sepsis). Es necesario la realización de pruebas de imagen. En todo diabético que presente fiebre, dolor abdominal, inflamación, en flanco, etc. con lentitud en la respuesta a antibióticos, es mandatorio la realización de una radiografía simple de aparato urinario, para valorar la existencia de gas perinefrítico o intraparenquimatoso. La imagen típica consiste en un halo de aire extraintestinal, en la zona de la silueta renal. Si persisten dudas habrá que realizar una T.A.C. la cual revelará la existencia de aire intraparenquimatoso o perinefrítico ${ }^{10,11}$. Otros métodos diagnósticos pueden ser la Urografía Intravenosa (U.I.V.) y la Ecografía. La U.I.V. suele mostrar además de los datos de la radiología simple, la anulación del riñón afecto, o la existencia de gas en la vía. La ecografía es una técnica altamente fiable en numerosas afecciones inflamatorias del riñón y suele ser la primera exploración después de la radiografía simple de abdomen, en el diagnóstico de infección urinaria con dolor abdominal, habiendo autores que la propugnan en el diagnóstico de la pielonefritis enfisematosa ${ }^{4}$. Sin embargo para otros su eficacia es dudosa debido a la existencia de gas que puede artefactar los hallazgos ${ }^{12}$. En cualquier caso la T.A.C. parece el método de imagen princeps. No sólo diagnostica claramente la existencia de gas intrarrenal sino que evalúa la cantidad de parénquima afectado, sirviendo para conocer la presumible funcionalidad de esa unidad renal, y planificar el eventual tratamiento quirúrgico ${ }^{2,4}$.

Debido a la amenaza vital que supone este cuadro es fundamental no sólo un rápido y certero diagnóstico, sino también un precoz manejo. $\mathrm{El}$ tratamiento debe iniciarse con un estricto control de la diabetes, la instauración de una pauta antibiótica correcta, empírica al inicio, cubriendo gram-negativos, y en espera del cultivo y antibiograma. La corrección de los trastornos hidroelectrolíticos, así como el mantenimiento de las constantes exigen el ingreso en una Unidad de Cuidados Intensivos. Dado que la mortalidad estimada para aquellos casos tratados médicamente es del $80 \%$, mientras que en los casos en los que se realiza tratamiento quirúrgico es del $30 \%$, la actitud agresiva es una máxima en el tratamiento de esta patología $2,7,13-15$. La mayoría de los autores proclaman la nefrectomía de urgencia como base del tratamiento quirúrgico. En ocasiones se ha planteado la posibilidad de colocación de una nefrostomía percutánea para derivar el contenido infeccioso y conseguir preservar esa unidad renal ${ }^{6,16,17}$. Nosotros pensamos que dada la gravedad del cuadro, y el hecho de que generalmente la destrucción del parénquima es masiva o submasiva y en cualquier caso existe casi siempre una microangiopatía de base, que hace que raramente ese riñón sea recuperable. Por otra parte el pus es demasiado viscoso para drenar por un catéter percutáneo además de que la naturaleza multiloculada de estas infecciones hacen que sea difícil su completa evacuación ${ }^{16}$. La única justificación para utilizar este procedimiento conservador sería la existencia de una pielonefritis bilateral (10\% de los casos), y el que sea un paciente monorreno. La preservación del parénquima renal es un importante objetivo que puede salvar la vida de estos pacientes ${ }^{18}$. 
A modo de conclusión podemos afirmar que el caso que presentamos reúne todas las características de un típico caso de pielonefritis enfisematosa. Lo único que no es típico es el sexo ya que es más frecuente en mujeres. El resto está en consonancia con toda la bibliografía revisada. Desde el modo de presentación, en un diabético mal controlado, con clínica característica, hasta los hallazgos radiológicos, la evolución, y el tratamiento instaurado. Para finalizar, nos parece de suma importancia recordar que: el diagnóstico tiene que ser sospechado, adelantándonos a los acontecimientos, el tratamiento con medidas de soporte en una unidad de Cuidados Intensivos y la antibioterapia agresiva son pilares fundamentales del diagnóstico, y que sobre todo el tratamiento quirúrgico agresivo con nefrectomía mejora ostensiblemente el pronóstico consiguiendo la supervivencia de los enfermos como es el caso del paciente expuesto en este artículo.

\section{REFERENCIAS}

1. KELLY HA, MAC CALLUM WG.: Pneumaturia. JAMA 1898; 31: 375.

2. SHOKEIR AA, EL-AZAB M, MOHSEN $\mathrm{T}$ et al.: Emphysematous pyelonefphritis: a 15-year experience with 20 cases. Urology 1997; 49: 343-346.

3. AHLERING TS, BOYD SD, HAMILTON CL et al.: Emphysematous pyelonephritis: a 5 year experience with 13 patients. J Urol 1985; 134: 1.086-1.088.

4. SCHAEFFER AJ: Infections of the urinary tract. In: Campbell's Urology. Eds: Walsh PC, Retik AB, Vaughan ED, Wein AS. Eighteen Edition. Philadelphia. Saunders Company 2002: 556-558.

5. MICHAELI J, MOGLE P, PERLBERGS et al.: Emphysematous pyelonephritis. J Urol 1984; 131: 203-208.

6. HUDSON MA, WEYMAN PJ, VAN DER VLIET AH et al.: Emphysematous pyelonephritis: successful management by percutaneous drainage. J Urol 1986; 136: 884-886.
7. BONSOMS N, MANCEBO J, BLANCH L et al.: Pielonefritis enfisematosa: aportación de un caso y revisión de la literatura. Rev Clin Esp 1992; 190: 72-74.

8. JOHNSON JR, IRETON RC, LIPSKY BA.: Emphysematous pyelonephritis caused by Candida Albicans. J Urol 1986; 136: 80-82.

9. LEVY AH, SCHWINGER HN.: Gas containing perinephritic abscess. Radiology 1953; 60: 720.

10. SHAHATTO N, AL AWADHI NZ, GHAZALI S.: Emphysematous pyelonephritis: surgical implications. Br J Urol 1990; 6: 572-574.

11. ELOUBEIDI MA, FOWLER VG Jr.: Images in clinical medicine. Emphysematous pyelonephritis. $N$ Engl J Med 1999; 341: 737.

12. RIBOT T, BONSOMS N, PRERA A et al.: Pielonefritis enfisematosa: una enfermedad que precisa un alto índice de sospecha para su diagnóstico precoz. Rev Clin Esp 1992; 191: 399.

13. SARMENTERO ORTIZ E, MORALES SORIANO R.: Pielonefritis enfisematosa. Actas Urol Esp 1994; 18: 836-837.

14. MONLLOR GISBERT J, GALBIS PALAU F.: Pielonefritis enfisematosa. Arch Esp Urol 1995; 48: 956-959.

15. VALBUENA ÁLVAREZ RJ, CAJIDE MONTERO JL, SANTOS-ASCARZ TABARES JL et al:: Pielonefritis enfisematosa. Actas Urol Esp 1997; 21: 154157.

16. KOH KB, LAM HS, LEE SH.: Emphysematous pyelonephritis: drainage or nephrectomy?. $\mathrm{Br} J$ Urol 1993; 71: 609-611.

17. CORR J, GLEESON M, WILSON G et al.: Percutaneous management of emphysematous pyelonephritis. Br J Urol 1993; 71: 487-488.

18. LOWE BA, POAGE MD.: Bilateral emphysematous pyelonephritis. Urology 1991; 37: 229-232.

Dr. A. Blanco Díez

Plaza Maestro Mateo, 9- 2o Izda.

15004 La Coruña

(Trabajo recibido el 10 enero 2003) 